\title{
DO WE NEED A NEW CLASSIFICATION OF AIRWAY DISEASES?
}

8

\section{THE CASE AGAINST}

Alvar Agusti ${ }^{1}$, Ian D Pavord ${ }^{2}$ Enfermedades Respiratorias, Spain 2. Respiratory Medicine Unit and Oxford NIHR Respiratory Biomedical Research Centre, Nuffield Department of Medicine, University of Oxford, Oxford, UK

Correspondence: Dr. Alvar Agusti, Respiratory Institute, Hospital Clinic. Villarroel 170, 08036 Barcelona. Tel: +3493 227 1701. E-mail: $\underline{\text { AAGUSTI@clinic.cat }}$

\section{Funding: none}

Key-words: Allergy, Asthma, Chronic Bronchitis, Emphysema, Precision medicine, Personalized medicine, Smoking, Taxonomy.

Words (excluding references): 913 words; References: 15; Tables: 0; Figures: 1. 
How do we classify human diseases (i.e., its taxonomy) is at the core of clinical practice because it guides their diagnosis and treatment ${ }^{1}$ The current classification of human diseases was proposed by Sir William Osler in the XIX century based on the main organ from which symptoms and signs originate and the histopathology findings in that organ. ${ }^{2}$ This classification has been (and still is) useful in clinical practice but has a number of important limitations ${ }^{3}$. First, most human diseases are still diagnosed (and treated) as if they were homogeneous entities, which is clearly not the case ${ }^{4}$. For instance, the chronic airway diseases, asthma and chronic obstructive pulmonary disease (COPD) are now recognized to be complex and often overlapping diseases, that require a much more detailed pathogenic characterization in order to provide the best possible treatment to an individual patient ${ }^{1}$. A second concern is that the Oslerian classification of diseases does not take into consideration susceptibility states or preclinical disease manifestations. For instance, since the landmark study of Fletcher and Peto it is clear that not all individuals are equally susceptible to the damaging effects of smoking ${ }^{5}$ and, more recently, it has become apparent that both asthma and COPD can originate very early in life. ${ }^{7-9}$ The need for a more specific approach to the diagnosis and, above all, treatment of chronic airway diseases has become clearly apparent in recent years, particularly as we enter a new era of "biologic treatment" of these diseases, which, hopefully, will lead to a much more precise clinical management of these patients ${ }^{1,10}$.

For these reasons, some authors advocate that we need a new classification of human diseases for the XXI century. ${ }^{3,4}$ Yet, it is not clear what this should look like. Loscalzo et al proposed that, in the post-genomic era, diseases need to be reclassified bottom-up 
44 (i.e., from genes to symptoms) in contrast to the top-bottom Oslerian classification,

which starts with their clinical presentation and then investigate the underlying biologic mechanisms to the extent possible ${ }^{3}$. They argue, compellingly, that the analysis of the complex molecular networks that underlie human diseases (i.e., network medicine) will help in this endeavour ${ }^{3,12}$. We fully agree that this strategy is likely to provide novel biological information, validate relevant biomarkers and provide novel therapeutic targets within a research framework. However, we doubt it will work in clinical practice. We argue that keeping the Oslerian classification, whose clinical utility has been clearly time-honoured, still provides the practicing clinician the necessary conceptual framework to establish an initial syndromic diagnosis from which to work up later the individual peculiarities of every patient ${ }^{13}$. In this context, the Oslerian diagnosis should be viewed as the starting point of the diagnostic process and not the final one.

Figure 1 compares the research and clinical practice approach to human diseases. From a research perspective, we can consider that the Exposome (i.e. the cumulative environmental exposures an individual encounters through life) interacts with the genetic background (Genome) of the patient through a number of multi-level, complex biological networks. As a result, one or more Endotypes (i.e., subtype of a disease defined functionally and pathologically by a molecular mechanism or by treatment response) might emerge or disappear dynamically through life, from pregnancy to death. The presence of these endotypes in any given individual may be revealed by one or more biomarkers (i.e., a characteristic that is objectively measured and evaluated as an indicator of normal biologic processes, pathogenic processes, or 
biological responses to a therapeutic intervention). Biomarkers do not necessarily need to be molecular markers. They can also be functional variables (e.g., lung function), imaging findings (e.g. presence of emphysema on computed tomography) or other clinically relevant measurements (please note that this is why biomarkers in

Figure 1 are now positioned in between the research and clinical practice environments).

From a clinical perspective, the diagnostic process began by the process of pattern recognition ${ }^{19}$. Indeed, physicians have long been trained to integrate different levels of potentially relevant information (symptoms, signs, results from both biological tests and imaging techniques, among others) to identify different clinical patterns which actually form the basis of the current Oslerian classification of diseases ${ }^{2}$. For the reasons argued above, we believe that this is a necessary but insufficient step in the clinical management of patients with chronic airway diseases. The specificity of this step can be augmented by the use of validated biomarkers (see above) and/or the recognition of a Clinical phenotype (i.e., a single or combination of disease attributes that describe differences between individuals as they relate to clinically meaningful outcomes ${ }^{20}$ ). Both lead to the final identification of so-called Treatable Traits (i.e., therapeutic target identified by "phenotype" or "endotype" recognition through validated biomarker(s) $\left.{ }^{10,21}\right)$. We think that this approach will combine "the best of two worlds", being of value toresearchers and clinicians. On the one hand, clinicians will not lose our rich heritage of pattern recognition in practice, which greatly helps to start (not finish) the diagnostic process. On the other, patients will benefit from a more precise management thanks to the advances made in research, which will hopefully 
92 help to estimate the risk-benefit ratio of any given treatment in an individual patient.

93 These treatable traits can easily be presented in the form of a "control panel" to the

94 practicing physician with the help of electronic medical records and artificial

95 intelligence approaches ${ }^{22}$.

96 
97

98

99

100

101

102

103

104

105

106

107

108

109

110

111

$1127 . \quad$ Lange $\mathrm{P}$, Celli B, Agusti A, et al. Lung-Function Trajectories Leading to Chronic

113 Obstructive Pulmonary Disease. New England Journal of Medicine 2015; 373(2): 111-

\section{REFERENCES}

1. Pavord ID, Beasley R, Agusti A, et al. After asthma - redefining airways diseases.

A Lancet commission Lancet 2017; 391(10118): 350-400.

2. Vanfleteren LEGW, Kocks JWH, Stone IS, et al. Moving from the Oslerian paradigm to the post-genomic era: are asthma and COPD outdated terms? Thorax 2014; 69(1): 72-9.

3. Loscalzo J, Kohane I, Barabasi AL. Human disease classification in the postgenomic era: a complex systems approach to human pathobiology. Mol Syst Biol 2007; 3: 124.

4. Kola I, Bell J. A call to reform the taxonomy of human disease. Nat Rev Drug Discov 2011; 10(9): 641-2.

5. Fletcher $\mathrm{C}$, Peto R. The natural history of chronic airflow obstruction. Br Med J 1977; 1(6077): 1645-8.

6. Martinez FD. Early-Life Origins of Chronic Obstructive Pulmonary Disease. New England Journal of Medicine 2016; 375(9): 871-8. 22. 
115 8. Agustí A, Noell G, Brugada J, Faner R. Lung function in early adulthood and

116 health in later life: a transgenerational cohort analysis. The Lancet Respiratory

117 Medicine 2017; 5(12): 935-45.

118 9. Agustí A, Bafadhel M, Beasley R, et al. Precision medicine in airway diseases:

119 moving to clinical practice. European Respiratory Journal 2017; 50(4): 1-13.

120 10. Barabasi AL, Gulbahce N, Loscalzo J. Network medicine: a network-based

121 approach to human disease. Nat Rev Genet 2011; 12(1): 56-68.

122 11. Celli BR, Agustí A. COPD: time to improve its taxonomy? ERJ Open Research $1232018 ; 4(1)$.

124 12. Agusti A. The disease model: implications for clinical practice. European 125 Respiratory Journal 2018; 51(4).

13. Han MK, Agusti A, Calverley PM, et al. Chronic Obstructive Pulmonary Disease

127 Phenotypes: The Future of COPD. American Journal of Respiratory and Critical Care Medicine 2010; 182(5): 598-604.

14. Agusti A, Bel E, Thomas M, et al. Treatable Traits: Toward Precision Medicine of 130 Airway Diseases. Eur Respir J 2016; 47: 410-9.

131 15. Agusti A, MacNee W. The COPD control panel: towards personalised medicine 132 in COPD. Thorax 2013; 68: 687-90. 
133 FIGURE LEGEND

134 Figure 1. Comparison of the research and clinical practice approaches to human

135 diseases. For further explanations, see text.

136 